\title{
The Specifics of Cartographic Semiology in Tactile Maps
}

\author{
Alena Vondrakova*, Radek Barvir, Jan Brus \\ ${ }^{a}$ Department of Geoinformatics, Faculty of Science, Palacký University Olomouc, 17. listopadu 50, Olomouc, Czechia \\ alena.vondrakova@upol.cz,radek.barvir@upol.cz,jan.brus@upol.cz.
}

* Corresponding author

Keywords: Tactile Maps, Cartography, Semiology, User Issues

\begin{abstract}
:
Spatial information became ordinary for everyday life, for example in different kinds of maps. The majority of maps are produced for reading with eyes. Nevertheless, people with visual impairment, including blind people, perceive the world differently and have an insufficient amount of possible ways how to investigate the surroundings.

Creating a tactile map for people with visual impairment and blind people is conceptually different, more expensive and more difficult comparing to normal maps. This is why researches in cartography focus the question how spatial information can be effectively presented to visually impaired people using modern technologies.

One of the solutions seems to be progress in modern 3D tactile-cartography linkable to the mobile device, such as smartphones and tablets. The modern mobile technologies with the internet connection and GNSS navigation brought new possibilities how to convert spatial information into voice quickly. Navigations use vibrations to provide the actual information, and other technologies help to make the 3D presentations of the geospace more accessible.
\end{abstract}

At the Department of Geoinformatics, Faculty of Science, Palacký University Olomouc, Czechia, several tactile maps were produced under the leadership of Vit Vozenilek, both interactive and non-interactive ones.

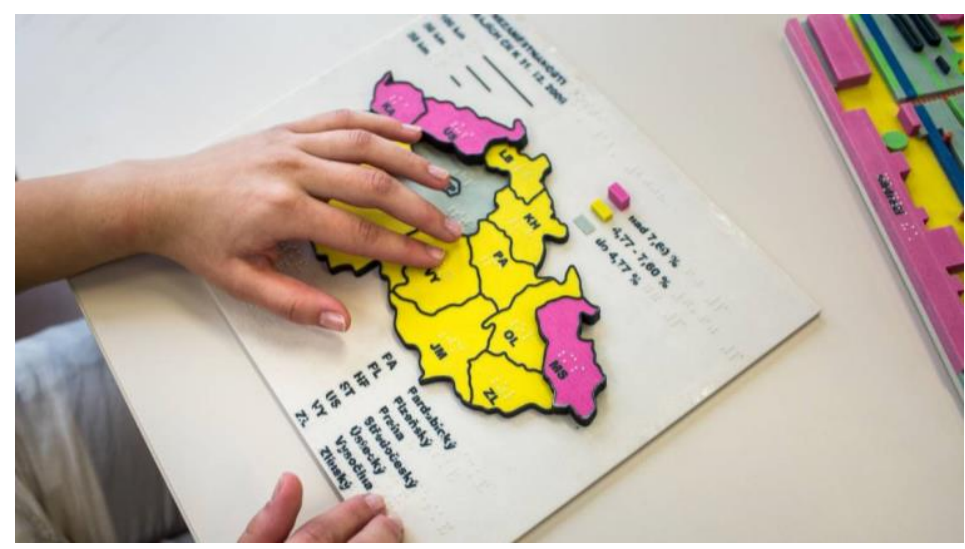

Figure 1. Example of non-interactive coloured 3D printed map with the presentation of the unemployment in the Czech Republic as the equivalent to the choropleth map in the traditional cartography.
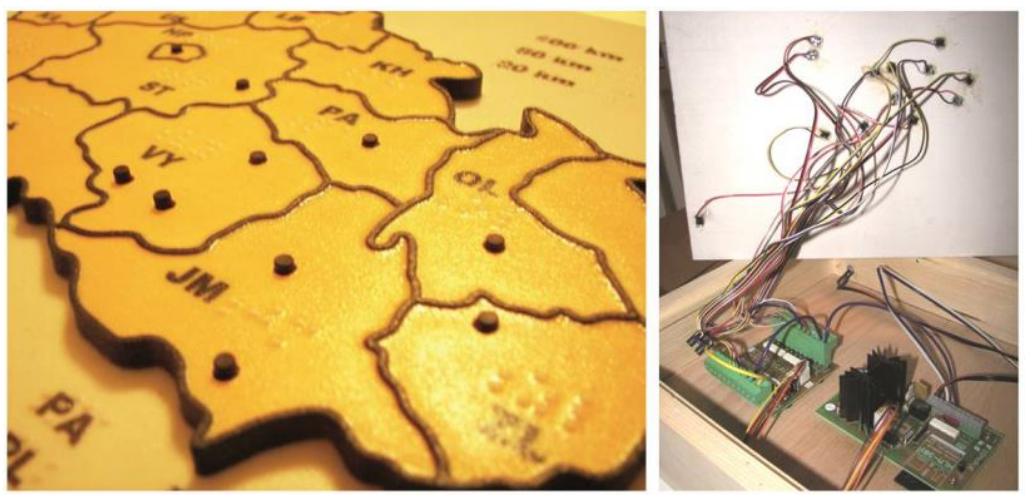

Figure 2. Example of interactive 3D tactile map developed by the research team at the Department of Geoinformatics, Faculty of Science, Palacký University Olomouc, using the touch-sensors situated in the map, and the sound-module to make the tactile map interactive. The user can gain a significantly larger amount of information from such type of the tactile map. 
Cartographic semiology deals with a theory of cartographic symbols and their use. This theory is based primarily on the general semiology (the science of symbols), theoretical cartography, information theory, cybernetics and engineering psychology. To the various disciplines of semiology belong semantics, which represents the relation between the map symbol to the content what it means, sigmatics, which defines the relationship of map symbols to the function expressed in terms of real content, syntactic, which describes the interactions of map symbols, grammar, that deals with the composition rule and specifies the map symbol to the system, and pragmatics, which describes the relationship of users to the map symbol system. It is necessary to design and apply specific methods of cartographic visualization that will be suitable for persons who are blind or have a serious visual impairment. Therefore, there is a significant need for adaptation to the target user needs.

Analogically to the traditional cartography, the fundamental unit of tactile cartographic semiology is a tactile map symbol. Comparing to conventional map symbol the tactile map symbols have an extra specific 3D features, including the vertical dimension, roughness and texture. The specific design of the tactile map symbol depends on the applied technology special tactile paper printers, plastic foils, metal engravings or modern type of 3D printing technology. Characteristics of tactile map symbols are used with regard to the possibilities of these technologies as well as in relation to the needs of the target group of users.

Within the project Perception of the geospace by the modern type of tactile maps the sampler designed characters by 51 respondents (31 blind persons and 20 persons with hard visually impairments) was tested. There were examples of different lines, different textures and point map symbols (Fig. 3). Part of these symbols was identified by respondents as most satisfactory, some of the characters were identified as unsatisfactory. These were mainly badly recognisable structures, lines unrecognisable by touch, confusing dotting, etc.

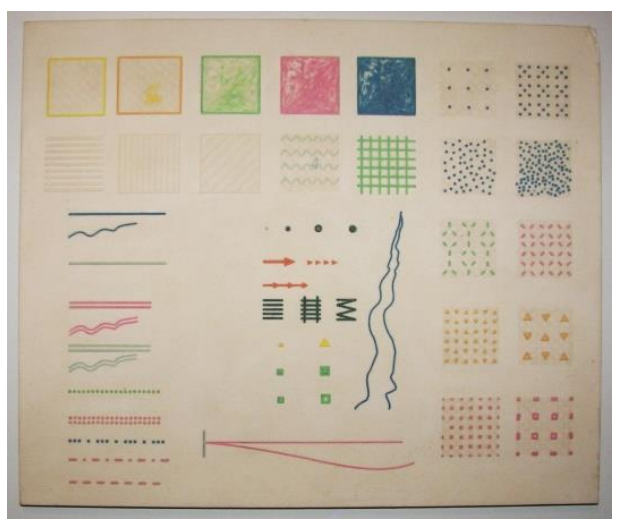

Figure 3. The sampler of map symbols for 3D printing. The suitability of each character was tested on a representative sample of users before implementation to the tactile map creation.

During the implementation of the project Development of independent movement through tactile-auditory aids, the gained knowledge was applied to the production of modern, tactile maps linked to the mobile technology (smartphone, tablet, etc.). Because the modern tactile maps using TouchIt3D technology (Barvir, 2017; Barvir et al., 2018) require many different sizes of 3D map objects, also the map symbols have to be different.

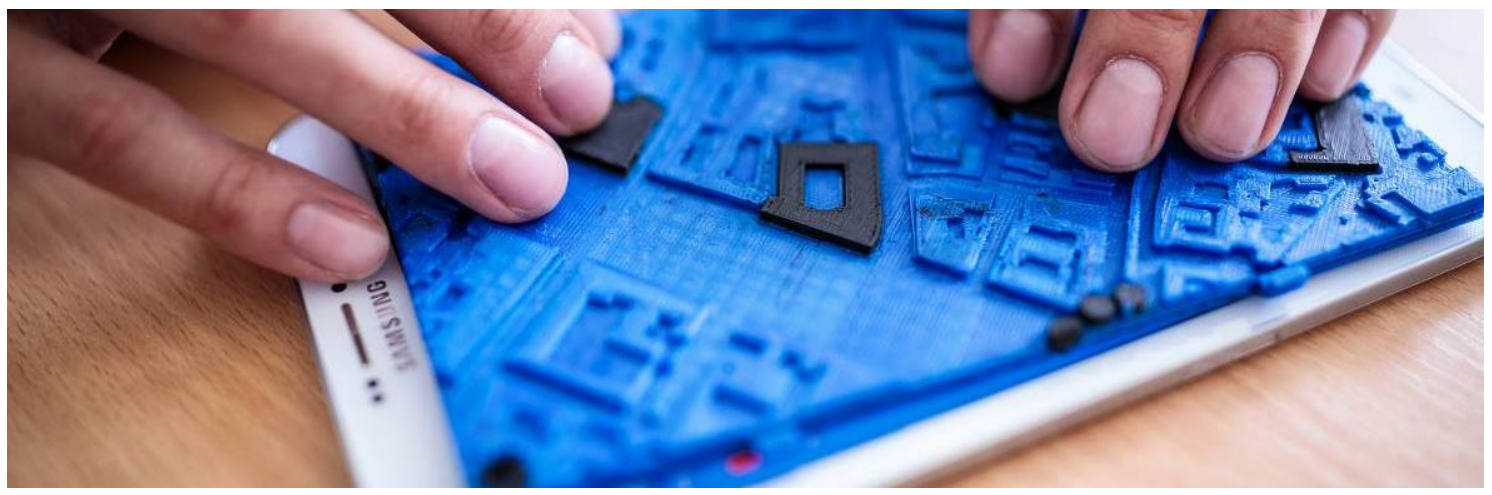

Figure 4. One of the first prototypes of a tactile map using TouchIt3D technology.

Preliminary results of the user testing provide new information about map symbol perception by people with visual impairment, using a new type of 3D tactile maps created with TouchIt3D technology. Testing and prototyping are ongoing, and the conference contribution will bring the latest research results.

This research is implemented within the project Development of independent movement through tactile-auditory aids, Nr. TL01000507, supported by the Technology Agency of the Czech Republic. 